\title{
Evaluación del aprendizaje de la estadística orientada a proyectos en estudiantes de ingeniería
}

\section{Evaluation of the learning of the statistics oriented to projects in engineering students}

\author{
Hugo Alejandro Alvarado Martínez ${ }^{1}$ \\ Maritza Katherine Galindo Illanes ${ }^{2}$ \\ María Lidia Retamal Pérez ${ }^{3}$
}

\begin{abstract}
Resumen: En este trabajo se evalúa el aprendizaje de conceptos y procedimientos estadísticos descriptivos mediante la estrategia basada en proyectos. Apropiándonos de la guía de análisis del componente de idoneidad didáctica, se examinan los niveles alcanzados sobre estadística descriptiva por un grupo de 125 estudiantes de ingeniería. Los resultados de aprendizaje indican que un grupo importante de estudiantes logró aplicar correctamente los procedimientos de la estadística descriptiva y utilizar los recursos informáticos en el análisis de información. Sin embargo, hubo dificultades en la declaración de los objetivos, tabulación de datos en una y dos variables, alcanzando el nivel más bajo lectura e interpretación de gráficos, relacionar medidas estadísticas y determinar relaciones entre variables. La metodología utilizada acrecentó la motivación, autonomía y aplicación de estrategias en el análisis de los datos para la solución de situaciones reales.
\end{abstract}

Palabras clave: Estadística, aprendizaje, proyectos, educación universitaria, ingeniería.

Fecha de recepción: 15 de mayo de 2017. Fecha de aceptación: 12 de julio de 2018

1 Universidad Católica de la Santísima Concepción, Chile, alvaradomartinez@ucsc.cl, orcid.org/ 00000002-3729-3631

2 Universidad San Sebastián, Chile, maritza.galindo@uss.cl, orcid.org/0000-0003-1394-2075

3 Universidad Católica de la Santísima Concepción, Chile, Iretamal@ucsc.cl, orcid.org/0000-0002-6696-0077 


\begin{abstract}
This work is evaluating learning concepts and descriptive statistical procedures through the strategy based on projects. Appropriating the analysis guide of the component of didactic suitability, the levels reached on descriptive statistics were examined by a group of 125 engineering students. The learning results indicate that an important group of students managed to apply the descriptive statistics procedures correctly and use the computer resources in the information analysis. However, there were difficulties in the declaration of objectives, tabulation data in one and two variables, reaching the lowest level reading and interpretation of graphs, relating statistical measures, and determining relationships between variables. The methodology used increased the motivation, autonomy and application of strategies in the analysis of the data for the solution of real situations.
\end{abstract}

Keywords: Statistics, learning, project, higher education, engineering.

\title{
INTRODUCCIÓN
}

La estadística puede ser considerada, en el currículo de ingeniería, como una etapa de transición entre los conocimientos de matemáticas y ciencias de la ingeniería (Retamal, Alvarado y Rebolledo, 2007), y en la práctica profesional como la ciencia de los datos, siendo importante las aplicaciones del razonamiento estadístico en situaciones tales como: el estudio de caudales de ríos para la construcción de puentes; estudio de olas para el diseño de un puerto marítimo y el análisis del procesamiento de datos para la toma de decisiones.

Las escuelas de ingeniería están interesadas en estructurar sus carreras orientadas al desarrollo de competencias (Letelier, López, Carrasco y Pérez, 2005), promoviendo un cambio profundo en la readecuación del contenido disciplinario en las ciencias básicas y en la evaluación formativa orientada hacia los resultados de aprendizaje. Esto conlleva el desafío de articular las ciencias básicas y ciencias de la ingeniería, favoreciendo el desarrollo de las competencias profesionales y la formación estadística del ingeniero. No obstante, la asignatura de Estadística es considerada difícil para los estudiantes de ingeniería (Alvarado y Batanero, 2007), y los nuevos planes de estudio no promueven la comprensión exhaustiva de conceptos básicos como la media aritmética y varianza, descuidando las consecuencias que puede tener en el aprendizaje de tópicos más 
avanzados. Concordamos con Chance, delMas y Garfield (2004) acerca de la importancia de apropiarse de conceptos y procedimientos previos de la inferencia estadística, tales como el análisis de gráficos, las medidas de tendencia central y de dispersión.

En situaciones-problemas de ingeniería la estadística descriptiva es la primera fase de exploración de los datos, proporcionando una metodología para analizar la variabilidad y determinar relaciones entre las variables estadísticas. Es un tópico de la estadística que conjuga muchos conceptos asociados, diversos tipos de lenguaje y representaciones, propiedades, procedimientos y argumentos. Sin embargo, se reporta una comprensión superficial de conceptos estadísticos básicos; sobre representaciones gráficas y medidas estadísticas (Juárez e Inzunsa, 2014), la mediana (Mayén, Batanero y Díaz, 2009), interpretación de los coeficientes de correlación y determinación (Inzunza, 2016) y procesos estadísticos (Chance, delMas y Garfield, 2004; Ben-Zvi y Garfield, 2004).

Las orientaciones sobre la enseñanza de la estadística indican que desde temprana edad se debe desarrollar el sentido estadístico, y cuya efectividad en los estudiantes se favorece con una enseñanza basada en investigaciones y proyectos (Batanero, Díaz, Contreras y Roa, 2013; Flores y Pinto, 2017). En el nivel universitario los estudiantes no logran comprender las principales ideas estadísticas y aplicar el razonamiento estadístico en situaciones contextualizadas (Alvarado, Galindo y Retamal, 2013). Coincidimos con los autores en hacernos cargo del problema didáctico sobre la transmisión de una estadística sin sentido a los estudiantes.

El objetivo de este trabajo es evaluar el aprendizaje orientado a proyectos sobre estadística descriptiva por un grupo de estudiantes de ingeniería civil. Se utiliza como procedimiento la guía de análisis de la componente de idoneidad didáctica propuesta por Godino (2009) y la valoración de los niveles de los descriptores de dicha componente por Arteaga, Batanero, Cañadas y Gea (2012).

\section{MARCO DE REFERENCIA}

\section{SENTIDO ESTADístico Y SU DESARROLLO}

Se concibe el sentido estadístico como unión de la cultura estadística (Watson, 2006) y razonamiento estadístico (Wild y Pfannkuch, 1999). La cultura estadística considera la componente de la apropiación correcta de las ideas fundamentales 
de datos y gráficos (Burrill y Biehler, 2011; Batanero, Díaz, Contreras y Roa, 2013;

Sánchez y Hoyos, 2013), y para desarrollar el razonamiento estadístico se sugiere implementar en el aula de estadística el trabajo con proyectos (Batanero, Burrill y Reading, 2011; MacGillivray y Pereira-Mendoza, 2011). En particular, tendremos en cuenta el pensamiento estadístico que conjuga el conocimiento estadístico, el conocimiento del contexto y la información contenida en los datos (Wild y Pfannkuch (1999).

\section{APRENDIZAJE DE LA ESTADÍSTICA CON PROYECTOS EN INGENIERÍA}

Batanero, Díaz, Contreras y Roa (2013) sugieren que la mejor forma de ayudar a los estudiantes a desarrollar su sentido estadístico en el aula es mediante el trabajo con proyectos, planteados por el profesor o escogidos libremente por los estudiantes. Gil (2010: 122), define:

el trabajo por proyectos como una propuesta didáctica que comprende un conjunto de tareas, organizadas y secuenciadas, llevadas a cabo mediante el trabajo colaborativo con el objetivo de obtener un resultado o producto determinado.

Esta metodología considera las diferentes fases de una investigación estadística: planteamiento de un problema, decisión sobre la información, recogida y análisis de datos y obtención de conclusiones sobre el problema planteado (Batanero y Díaz, 2011). Wild y Pfannkuch (1999) hacen notar como elementos básicos de razonamiento estadístico el reconocer la necesidad de los datos y la integración de la estadística y el contexto.

En la actualidad, las Escuelas de Ingeniería están presentando nuevos modelos educativos basados en competencias; destacando el rol activo del estudiante, la utilización de recursos informáticos y de plataformas virtuales de aprendizaje en la docencia, la reflexión sobre la retroalimentación oportuna durante el proceso de la evaluación de los resultados de aprendizaje y la preparación del profesorado en metodologías de enseñanza actualizada. En consecuencia, la tendencia es implementar un currículo menos técnico y cada vez más práctico, en un contexto de Concebir-Diseñar-Implementar-Operar (CDIO, http://www.cdio. org/), siendo uno de los estándares los resultados de aprendizaje (Muñoz, Martínez, Cárdenas y Cepeda, 2013). Esto ha suscitado un interés sobre la formación estadística de los ingenieros (Martín, 2006; Romeu, 2006). 
Este trabajo, en correspondencia con los estándares CDIO, considera dos resultados de aprendizaje, RA1: Utilizar los elementos metodológicos de la estadística descriptiva en una y dos variables en la descripción e interpretación de datos y elaboración de informes relacionada con la ciencia de la ingeniería, RA2: Uso de recursos informáticos en el análisis de información estadística para la elaboración de informes orientados a proyectos.

\section{INVESTIGACIONES PREVIAS SOBRE ESTADÍSTICA DESCRIPTIVA}

La comprensión de información estadística involucra habilidades básicas como la capacidad de organizar datos, construir y presentar tablas, y reconocer e interpretar diferentes representaciones de datos (Ben-Zvi y Garfield, 2004; Estrella y Vidal-Szabó, 2017). Juárez e Inzunsa (2014) en un estudio con profesores de matemáticas detectaron un razonamiento de nivel bajo sobre conceptos estadísticos básicos; una comprensión superficial sobre la interpretación y conversión de representaciones gráficas, las medidas de tendencia central y de variabilidad. Además, utilizan pocos conceptos para describir y comparar distribuciones de datos. Aunque los estudiantes realizan bien los cálculos no los interpretan adecuadamente y carecen de comprensión del proceso estadístico (Chance, delMas y Garfield, 2004). Más aún, Ben-Zvi y Garfield (2004) señalan que los estudiantes se conforman con dar una respuesta en problemas contextualizados en lugar de elegir un procedimiento estadístico pertinente.

La literatura pone en evidencia la complejidad de la comprensión de las medidas de posición central, por ejemplo en la construcción progresiva de su significado. Encontramos investigaciones acerca del significado de la media aritmética en libro de texto y de conceptos relacionados a ella, que deben tenerse presente para el análisis de los errores y dificultades en lo procedimental y conceptual en otros tópicos de introducción a la inferencia estadística (Cobo y Batanero, 2004; Alvarado y Segura, 2012). Investigaciones sobre dificultades de comprensión de la mediana, al no percibir que puede ser mejor representante de posición del conjunto de datos según los contextos, se manifiestan en estudiantes de secundaria y bachillerato (Carvalho, 2001; Mayen, Cobo, Batanero y Balderas, 2007; Mayen, Batanero y Díaz, 2009), en futuros profesores de educación primaria (Groth y Bergner, 2006).

Inzunza (2016) señala que la comprensión de la correlación y la regresión lineal requiere del dominio de una estructura de una relación bivariada e 
interés en la construcción de modelos y en la predicción de variables. En su investigación con 34 estudiantes de informática (18-19 años) enfatiza la dificultad para distinguir la variable respuesta de la variable explicativa para la comprensión de la regresión. Otros estudios del tema citados por el autor analizan las estrategias de estimación de la correlación en los diagramas de dispersión y desarrollo del razonamiento correlacional a través de la enseñanza.

Si bien se reportan deficiencias sobre la comprensión de medidas de posición central, representaciones gráficas y asociación de variables, son escasas las investigaciones en el nivel universitario de ingeniería.

\section{COMPONENTES DE IDONEIDAD DIDÁCTICA}

De acuerdo al marco teórico del Enfoque Ontosemiótico tendremos en consideración las diferentes entidades primarias como constituyentes del significado de un objeto matemático (por ejemplo, estadística descriptiva), que son las que se analizan en este trabajo: Situaciones-problema que inducen actividades matemáticas y definen el campo de problemas asociado al objeto; lenguaje y representaciones materiales utilizadas en la actividad de resolución de problemas (términos, expresiones, símbolos, tablas, gráficos); reglas que utiliza un sujeto cuando se enfrenta a un problema e intenta de resolverlo mediante procedimientos, algoritmos y operaciones; argumentos y demostraciones que empleamos para probar sus propiedades y que llegan a formar parte de su significado; relaciones y conexiones entre los objetos matemáticos (Godino y Batanero, 1997).

En este marco se define la idoneidad epistémica como una de las seis componentes de idoneidad didáctica que valora el grado de representatividad de los significados institucionales implementados (o pretendidos), respecto de un significado de referencia (Godino, Giacomone, Batanero y Font, 2017).

En este estudio, se utilizará la pauta de análisis de idoneidad didáctica (Godino, 2009), para evaluar la componente de idoneidad epistémica mediante niveles de observación para analizar los descriptores sobre estadística descriptiva (Arteaga, Batanero, Cañadas y Gea, 2012). 


\section{METODOLOGÍA}

\section{MUESTRA}

Participaron en la investigación 125 estudiantes universitarios de ingeniería (19-20 años), de las especialidades de ingeniería civil industrial 89 (71,2\%) y civil geológica 36 (28,8\%). De los 125 estudiantes 45 (36\%) son mujeres. De acuerdo al plan de estudios los estudiantes estaban cursando segundo año académico y les correspondía iniciar la asignatura semestral de probabilidades y estadística de su malla. De los 125 estudiantes 25 (20\%) eran de tercer año quienes estaban llevando el curso por más de una oportunidad.

El programa de actividad curricular comprendía a la semana tres horas de cátedra, una hora de práctica y una hora de laboratorio de computación en la que utilizaron la planilla Excel y el programa R. El curso lo componían cuatro profesores de cátedra, quienes tienen más de una década de experiencia en la enseñanza de la Estadística en el nivel universitario. También, las actividades de aprendizaje prácticas fueron apoyadas por otros cuatro profesores con formación estadística en las sesiones de ayudantía y laboratorio de computación.

La inscripción de los estudiantes en las cuatro secciones disponibles del curso la realizaron por internet (requisito es haber aprobado el curso de cálculo II), de manera que una vez completada una sección debe incorporarse a la que está con cupos disponibles. En consecuencia, el curso contempló cuatro secciones de 46, 35, 24 y 20 estudiantes de las dos especialidades.

\section{DESARROLLO DE LA ENSEÑANZA}

La secuencia de aprendizaje de la estadística orientada a proyectos incluía la primera unidad del curso, estadística descriptiva en una y dos variables, la que se realizó con actividades diferenciadas y desarrolladas en grupos de al menos dos estudiantes fuera clase en un tiempo de cuatro semanas. La planificación consideró las siguientes actividades asociadas según los resultados de aprendizaje:

a) discusión sobre el uso de la estadística descriptiva en situaciones propias de la ingeniería, resolución de problemas de forma individual y/o grupal, observación y tutoría de los talleres realizados en el aula y laboratorio de 
computación, revisión de apuntes de clases disponible en la plataforma virtual de la asignatura (RA1).

b) tutorías de uso de la planilla Excel y del software estadístico programa $\mathrm{R}$ para el análisis de datos y solución de pequeñas investigaciones en el contexto de la ingeniería (RA2).

\section{ACTIVIDADES PREVIAS DE INICIACIÓN CIENTÍFICA}

Con el propósito de socializar con los estudiantes los resultados de aprendizaje, el procedimiento de la estadística descriptiva y poner en juego las competencias de la cultura estadística de desarrollar la capacidad para interpretar y evaluar críticamente la información estadística y de discutir o comunicar las opiniones en diversos contextos (Gal, 2002: 2-3), los estudiantes trabajaron en el aula y laboratorio de computación dos actividades previas al proyecto:

- Estadística descriptiva en los medios. Buscar información acerca de la presencia del uso de la estadística descriptiva en los medios de comunicación; identificando, comentando y valorando los términos y conceptos presentes en noticias breves.

En la Tabla 1 se presenta los resultados obtenidos del curso conformado en grupos de dos o tres estudiantes. La tabulación se realizó mediante la lista de cotejo, con presencia o ausencia en los ítems. Se presentaron 55 trabajos; 34 por estudiantes de ingeniería civil industrial y 21 de ingeniería civil geológica. Se observa que $85 \%$ de los estudiantes buscó información en los medios de comunicación. En cambio, sólo 31\% de ellos realizó comentarios sobre las medidas estadísticas (media, mediana, moda). Los temas escogidos fueron en las áreas de la ingeniería, ámbito social, económico, deporte y medio ambiente. 
Tabla 1. Resultados sobre la presencia de la estadística en los medios

\begin{tabular}{l|c|c}
\hline \multicolumn{1}{c|}{ Criterio: Lista de cotejo } & Frecuencia éxito & Porcentaje \\
\hline $\begin{array}{l}1.1 \text { Búsqueda de información de la presencia de la estadística } \\
\text { en los medios de comunicación }\end{array}$ & 47 & 85 \\
\hline 1.2 Identifica conceptos básicos vistos en clase & 39 & 71 \\
\hline $\begin{array}{l}1.3 \text { Comenta sobre medidas estadísticas, representaciones } \\
\text { tabular y gráfica en las noticias }\end{array}$ & 17 & 31 \\
\hline 1.4 Concluye sobre la importancia de los datos en un contexto social & 24 & 44 \\
\hline
\end{tabular}

El docente analizó los resultados en conjunto con los estudiantes destacando los siguientes elementos: a) La observación de los múltiples usos de la estadística descriptiva en una y dos variables en los distintos ámbitos, del entorno social y profesional, b) el análisis de los distintos enunciados presentes en las noticias de actualidad, anuncios que cualquier ciudadano debe leer y entender con información numérica y gráfica, c) seleccionamos algunos trabajos para comentar cómo se presentan los datos y los distintos conceptos estadísticos y gráficos encontrados en los medios de comunicación. Un ejemplo se muestra en la Figura 1 en el ámbito político de interés por un grupo de estudiantes.

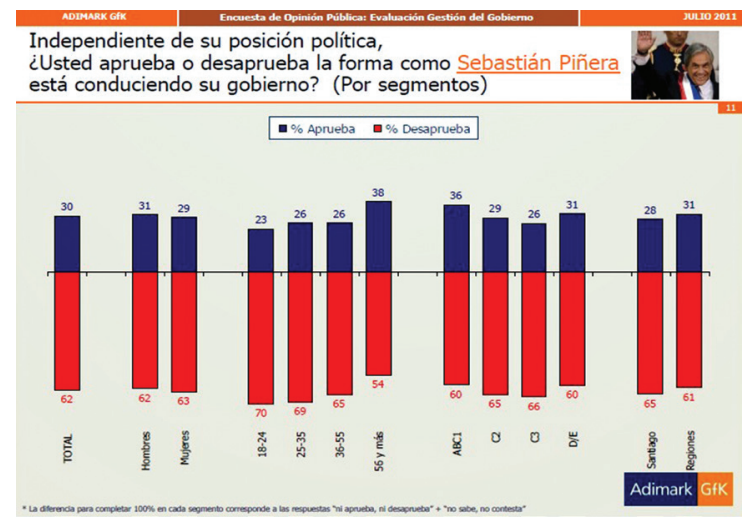

Figura 1. Evaluación de la gestión del gobierno

- Estadística descriptiva en un contexto social. Elaborar e interpretar información estadística de interés social de los jóvenes universitarios, como un primer acercamiento a la exploración de datos. 
Esta actividad permitió a los estudiantes poner a prueba sus conocimientos en proceso de estadística descriptiva, y a los docentes llevar a cabo una retroalimentación oportuna acerca de las dificultades encontradas en el procedimiento estadístico. Se presentaron 15 trabajos; por ejemplo un grupo conformado de 5 integrantes analizó una encuesta aplicada a 300 estudiantes de la universidad sobre el tiempo dedicado al facebook y a preparar sus evaluaciones (ver Figura 2, subida a la plataforma virtual del curso en formato power point).

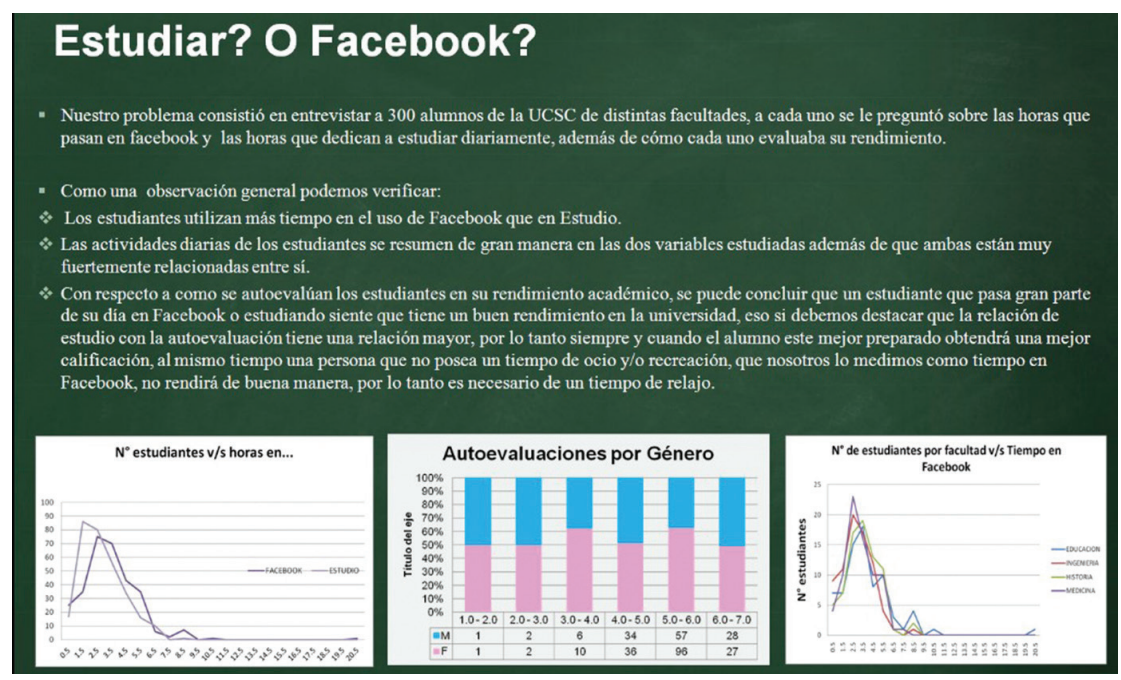

Figura 2. Distribución del tiempo a estudiar y revisar Facebook

El profesor realizó alcances a modo de retroalimentación, tales como: Se presentaron datos de encuestas con estudiantes de varias facultades de la universidad y tabuladas por género. Sin embargo, no se discutieron y analizaron las variables de estudio cruzando la información con las variables género y facultades. Hubo confusión en la simbología de las distribuciones de frecuencias, no se interpretaron los coeficientes de simetría, curtosis y gráficos. Las tablas de doble entrada se presentaron con muchos ceros en las celdas lo que no es recomendable. También, se presentaron trabajos que no exhibieron la base de datos con lo cual es difícil verificar su correcta tabulación de la distribución de frecuencias. 


\section{INSTRUMENTOS DE RECOLECCIÓN DE LA INFORMACIÓN}

Se utilizaron dos instrumentos de recolección de datos para la evaluación del aprendizaje basado en proyectos de ingeniería.

Los proyectos se analizaron por medio de la pauta de análisis de idoneidad didáctica (Godino, 2009), específicamente se aplicó la componente de idoneidad epistémica (ver Tabla 2).

Tabla 2. Descriptores de idoneidad epistémica en estadística

\begin{tabular}{l|l}
\hline \multicolumn{1}{c|}{ Componentes } & \multicolumn{1}{c}{ Descriptores } \\
\hline Situaciones-Problema & $\begin{array}{l}\text { P1. Se presenta una muestra representativa y articulada de situaciones } \\
\text { de contextualización, ejercitación y aplicación a las distintas } \\
\text { especialidades de la ingeniería. }\end{array}$ \\
\hline Lenguaje & $\begin{array}{l}\text { L1. Uso del lenguaje verbal y simbólico propio de la estadística. } \\
\text { L2. Construcción, lectura e interpretación de gráficos estadísticos. } \\
\text { L3. Se proponen actividades de expresión estadística e interpretación. }\end{array}$ \\
\hline Reglas (Definiciones, & $\begin{array}{l}\text { D1. Las Definiciones y propiedades son claras y correctas en este nivel } \\
\text { educativo. } \\
\text { p2. Se presentan los procedimientos fundamentales de la estadística } \\
\text { descriptiva. } \\
\text { D3. Se proponen situaciones que permitan generar definiciones, } \\
\text { propiedades y procedimientos. }\end{array}$ \\
\hline Argumentos & $\begin{array}{l}\text { A1. Las explicaciones, comprobaciones y demostraciones son adecuadas } \\
\text { al nivel educativo. } \\
\text { A2. Se promueven situaciones donde el estudiante tenga que } \\
\text { argumentar. }\end{array}$ \\
\hline Relaciones & $\begin{array}{l}\text { R1. Los objetos matemáticos (problemas, definiciones, propiedades,...) se } \\
\text { relacionan y conectan entre sí. }\end{array}$ \\
\hline
\end{tabular}

Arteaga, Batanero, Cañadas y Gea (2012) en su estudio de evaluación de conocimientos especializados de estadística en futuros profesores de educación básica presentaron cuatro niveles de observación para analizar los descriptores de la estadística descriptiva, y son los que se consideran en este trabajo (ver Tabla 3). 
Tabla 3. Niveles para evaluar descriptores de estadística descriptiva

\begin{tabular}{l|l}
\hline Nivel 0 & $\begin{array}{l}\text { No se observa el descriptor en el proyecto. Se deja la respuesta en blanco, no se } \\
\text { aplica en el análisis del proyecto. }\end{array}$ \\
\hline Nivel 1 & $\begin{array}{l}\text { Nivel elemental. El estudiante copia literalmente el descriptor sin indicar cómo lo } \\
\text { aplica. Reconoce su presencia pero no específica en qué modo se emplea dicho } \\
\text { descriptor. }\end{array}$ \\
\hline Nivel 2 & $\begin{array}{l}\text { Nivel intermedio. Aplica y hace referencia al descriptor pero sin centrarse en el } \\
\text { contenido estadístico. }\end{array}$ \\
\hline Nivel 3 & $\begin{array}{l}\text { Nivel avanzado. Aplica el descriptor a contenidos estadístico, referenciando diversos } \\
\text { tipos de lenguaje estadístico y capacidad de análisis de información. }\end{array}$ \\
\hline
\end{tabular}

También, se diseñaron dos instrumentos, una pauta de valoración de las comunicaciones de los trabajos presentados al finalizar el informe escrito (ver Tabla 11) y una pauta de autovaloración de los estudiantes según nivel de logro en los descriptores de un proyecto (ver Tabla 12).

\section{ANÁLISIS DE LOS RESULTADOS}

En lo que sigue se analiza los resultados obtenidos de los informes de proyectos situados en ingeniería por grupos de estudiantes. Por medio de una pauta general de elaboración de proyectos se solicitó a los estudiantes caracterizar y plantear un problema a la ingeniería mediante un informe orientado a proyectos escrito en Word, realizar la búsqueda sistemática de documentación, enunciar objetivos e hipótesis, utilizar el razonamiento estadístico, los recursos informáticos y discutir críticamente los resultados obtenidos.

A continuación, siguiendo la pauta de idoneidad epistémica y los niveles de los descriptores en estadística descriptiva, se resume los resultados para cada una de las componentes, mostrando algunos ejemplos de respuestas en los niveles 1, 2 y 3 . Se analizaron 45 trabajos entregados por 125 participantes; compuesto por 36 grupos de tres estudiantes, 8 grupos de dos estudiantes y un grupo de un integrante. 


\section{Situaciones-problemas}

La evaluación del descriptor P1: presentar una muestra representativa y articulada de situaciones de contextualización, ejercitación y aplicación a las distintas especialidades de la ingeniería, consideró tres aspectos como evidencia: el título del trabajo, la fundamentación del problema y declaración de las variables cuantitativas y cualitativas en estudio. En cuanto al título del proyecto contextualizado los estudiantes presentaron los siguientes:

Análisis estadístico químico del suelo en la ciudad de Arica - análisis hidrológico del caudal del río Laja - análisis químico de suelos de Chaitén y Santa Bárbara - el mito sobre las predicciones sismológicas - producción del cobre en Codelco - estudio de suelo - impacto vial en una arteria de Concepción distribución y consumo de bebidas gaseosas en el gran Concepción - estudio del desempeño de la locomoción colectiva - estudio de la telefonía móvil - distribución de energía eléctrica por clientes en Chile - ventas reales en tiendas VayVen - turismo en Chile - disponibilidad de materia prima en una empresa - pronóstico de exportación entre subproducto en el Bío Bío - estudio estadístico sobre las líneas de microbuses que conforman el sistema de transporte urbano de la ciudad de Concepción - estudio y análisis de las actividades económicas regionales y su relevancia a nivel país.

Hacemos notar, que hubo trabajos analizados con datos reales de hidrología y química de suelo, concedidos por docentes de la Facultad de Ingeniería. De esta manera, los estudiantes han iniciado el vínculo con problemas propios de ciencias de la ingeniería, lo que los hace protagonista de su propio aprendizaje.

Tabla 4. Frecuencia (y porcentaje) del nivel de aplicación del descriptor situación-problema

\begin{tabular}{c|c}
\hline & P1 \\
\hline Nivel 1 & $23(18,4 \%)$ \\
\hline Nivel 2 & $87(69,6 \%)$ \\
\hline Nivel 3 & $15(12,0 \%)$ \\
\hline
\end{tabular}

La Tabla 4 no muestra el nivel 0 que supone la no presencia del descriptor P1. $18,4 \%$ de los estudiantes presentaron un tema sin ahondar en su relevancia y pertinencia a la ingeniería; por ejemplo, el grupo A de estudiantes aborda el 
tema del consumo de bebidas gaseosas en Chile aplicando el descriptor P1 a nivel 1, mostrando una argumentación débil de contexto en la ingeniería.

Fundamentación: Los datos que quisimos recopilar lo hicimos pensando en la masiva influencia que tienen las bebidas gaseosas a lo largo de todo el mundo, en este caso nos centramos en la localidad de Concepción (Chile). Los datos obtenidos se refieren al consumo y precio de bebidas gaseosas no alcohólicas que tienen los integrantes por familia.

Variables: Número de personas en la familia, consumo de bebidas gaseosas en un día (litros) y gasto de dinero en las bebidas gaseosas.

69,6\% de los estudiantes contextualizó y fundamentó su problema. En los trabajos se argumentó la problemática con documentos disponibles en internet, en la biblioteca de la universidad, medios de comunicación y apoyo de profesores investigadores de la Facultad de Ingeniería. No obstante, si bien los estudiantes deben explicar, caracterizar y plantear adecuadamente un problema, no destacaron las herramientas estadísticas implícitas para su solución. Además, sólo $12 \%$ de los estudiantes argumentaron su situación de contexto, declarando correctamente las variables y objetivos propuestos. Por ejemplo, el grupo B estudió el consumo de luz en familias aplicando el descriptor P1 a nivel 3, denotando dos objetivos específicos que conlleva la síntesis de información y relacionar las variables presentes en el problema.

01. Describir el consumo promedio de energía eléctrica por familia que conforma la ciudad de Concepción.

O2. Analizar las relaciones que existen entre las variables "Consumo kWh por hogar/ Total Boleta (\$)", "Consumo kWh por hogar/ Cantidad Integrantes Grupo Familiar", "Consumo kWh por hogar/ electrodomésticos que más se Utilizan”, "Total Boleta (\$)/ Cantidad Integrantes, Grupo Familiar.

\section{Lenguaje}

Un segundo componente de idoneidad epistémica evaluada fue el lenguaje utilizado por los estudiantes. Se establecieron tres descriptores en el desarrollo del proyecto: 
L1. Uso del lenguaje verbal y simbólico propio de la estadística. En el proceso de recogida y disposición tabular de los datos la expectativa fue observar registrar la utilización de términos estadísticos en la elaboración de tablas de frecuencias. “Con frecuencia se emplean símbolos para referirse a la media, moda, mediana y rango. Asimismo se emplea el lenguaje numérico para expresar los datos y los cálculos de estadísticos" (Arteaga, Batanero, Cañadas y Gea, 2012). En la mayoría de los proyectos emergieron una variedad de términos, tales como: datos, variable estadística, distribución de frecuencias, clases individuales o por intervalos, datos no agrupados, simetría, valor medio, desviación estándar, cuartiles.

L2. Construcción, lectura e interpretación de gráficos estadísticos. Se observaron diferentes modos de expresión matemática (verbal, gráfica, simbólica...), traducciones y conversiones entre los mismos. Según Wild y Pfannkuch (1999) el lenguaje gráfico tiene un papel esencial en la organización, descripción y análisis de datos, al ser un instrumento de transnumeración. En particular, los estudiantes para resumir sus datos emplearon diversos tipos de gráficos, como diagramas de barras o líneas, histogramas, gráficos de puntos y gráficos de cajas (box plot).

L3. Se proponen actividades de expresión estadística e interpretación. Durante el avance de proceso se evaluaron situaciones de comunicación utilizando un lenguaje técnico y comprensible acorde al nivel educativo. Los tipos de comunicación que se consideraron fueron: la asistencia de los estudiantes en horario de consulta del docente, comunicación escrita en la entrega del informe y la discusión oral en la presentación de los trabajos.

Para evaluar el descriptor L3 se llevó un registro de asistencia de los estudiantes, obteniendo 76,8\% de consultas en oficina de los docentes, permitiendo dialogar y guiar la recogida de información, aunque la asistencia fue variada por los integrantes en cada grupo. 100\% de los estudiantes cumplieron con la entrega del informe escrito expresando una lectura comprensible de acuerdo a la pauta del informe del proyecto. También, 96\% de los estudiantes defendieron su proyecto en formato Power Point. 
Tabla 5. Frecuencia (\%) del nivel de aplicación y descriptores del lenguaje estadístico

\begin{tabular}{c|c|c}
\hline & L1 & L2 \\
\hline Nivel 1 & $49(39,2 \%)$ & $43(34,4 \%)$ \\
\hline Nivel 2 & $64(51,2 \%)$ & $74(59,2 \%)$ \\
\hline Nivel 3 & $12(9,6 \%)$ & $8(6,4 \%)$ \\
\hline
\end{tabular}

La Tabla 5 presenta los resultados de aplicación de los descriptores L1 y L2, hacemos notar que no se observó el nivel 0. De los estudiantes, 39,2\%, mostró un número determinado de conceptos de estadística descriptiva disponible en el resumen de estadísticos por Excel (L1 a nivel 1). Mediante el resumen estadístico mostrados en los trabajos 51,2\% del grupo curso realizó interpretaciones técnicas de las medidas estadísticas sin compararlas entre ellas y relacionarlas con su problemática. Por ejemplo, el grupo C aplica el descriptor L1 a nivel 2 a una situación que no es del ámbito de la ingeniería, evidenciando el resumen estadístico de las ventas de 113 fármacos seleccionados de una farmacia sin relacionar medidas estadísticas o interpretaciones descontextualizadas.

Figura 3. Resumen estadístico sobre ventas de fármacos

\begin{tabular}{|c|c|c|}
\hline & & \multirow{6}{*}{$\begin{array}{l}\text { Interpretación: } \\
\text { Dentro de los datos que se presentan podemos ver } \\
\text { que el precio promedio de toda la muestra de } \\
\text { medicamentos presentados es de } \$ 1389,64 \text { y el dato } \\
\text { que divide la muestra por la mitad es de } \$ 1080 \text {. } \\
\text { El valor de fármacos que más se repite es de } \$ 630 \text { el } \\
\text { cual está bajo el valor promedio de los medicamentos. }\end{array}$} \\
\hline Media & 1389,646018 & \\
\hline Error típico & 102,9465489 & \\
\hline Mediana & 1080 & \\
\hline Moda & 630 & \\
\hline $\begin{array}{l}\text { Desviación } \\
\text { estándar }\end{array}$ & 1094,336826 & \\
\hline
\end{tabular}

También, es preocupante que sólo 9,6\% de los estudiantes utiliza correctamente el lenguaje verbal y simbólico de la estadística descriptiva (L1 nivel 3). Un ejemplo es proporcionado de forma correcta por el grupo D sobre el tema del consumo de agua por vivienda en $\mathrm{m}^{3}$ de 200 casas en el cual relacionan e interpretan las medidas de tendencia central mediante datos no agrupados. 
Figura 4. Resumen estadístico sobre consumo de agua

\begin{tabular}{|c|c|c|}
\hline \multicolumn{2}{|l|}{ Resumen estadístico } & \multirow{11}{*}{$\begin{array}{l}\text { Interpretación: } \\
\text { Media: el promedio consumido de agua por casa } \\
\text { es de } 38,025 \text { (m3). } \\
\text { Mediana: el 50\% de las casas consume a lo más } \\
37(\mathrm{~m} 3) \text { de agua. } \\
\text { Moda: la mayoría de las casas consume } 18(\mathrm{~m} 3) \text { de } \\
\text { agua. } \\
\text { Desviación estándar: las casas tienen una } \\
\text { desviación promedio de agua de } 15,346 \text { (m3) con } \\
\text { respecto a la media. } \\
\text { Curtosis: K=-3.42844196, k<0... los valores anteriores } \\
\text { implican que la distribución es platicúrtica. } \\
\text { Como la media es mayor que la mediana y moda } \\
\text { la distribución del consumo de agua es asimétrica } \\
\text { positiva. }\end{array}$} \\
\hline Agua (m3) & & \\
\hline Media & 38.025 & \\
\hline Error típico & 1.08515422 & \\
\hline Mediana & 37 & \\
\hline Moda & & \\
\hline Desviación estándar & 15.3463981 & \\
\hline $\begin{array}{l}\text { Varianza de la } \\
\text { muestra }\end{array}$ & 235.511935 & \\
\hline Curtosis & -0.42844196 & \\
\hline $\begin{array}{l}\text { Coeficiente de } \\
\text { asimetría }\end{array}$ & 0.54309179 & \\
\hline Cuenta & 200 & \\
\hline
\end{tabular}

En cuanto al descriptor L2 la elaboración e interpretación de gráficos estadísticos, se observa en la Tabla 5 que 34,4\% del grupo curso presentó varios gráficos obtenidos de la planilla Excel; 59,2\%, de los estudiantes, llevó a cabo interpretaciones básicas de gráficos y sólo 6,4\% elaboró e interpretó de forma correcta los gráficos en su contexto, lo cual es preocupante en este nivel educativo. Un ejemplo, llevado a cabo por el grupo D, utilizó el gráfico circular para el consumo de agua en $\mathrm{m}^{3}$ en una muestra de viviendas de Concepción a partir de la representación tabular de datos agrupado por intervalo de clases. Lo correcto es representar la información mediante un histograma de frecuencias para variables de tipo continua.

Aquí está nuestra tabla para el consumo de agua por vivienda, en $\mathrm{m}^{3}$. Elegimos una distribución de datos por intervalos, al ser una variable cuantitativa discreta. También se muestra el gráfico circular respectivo (Tabla 6) 
Tabla 6. Representación tabular y gráfica

\begin{tabular}{|r|r|r|c|r|r||}
\hline Agua $\mathrm{m}^{3}$ & $\mathrm{ni}$ & $\mathrm{Ni}$ & \multicolumn{1}{c|}{$\mathrm{fi}$} & \multicolumn{1}{c|}{$\mathrm{Fi}$} & $\mathrm{mi}$ \\
\hline$[16-24]$ & 43 & 43 & $21.5 \%$ & $21.50 \%$ & 20 \\
\hline$[24-32]$ & 37 & 80 & $18.5 \%$ & $40 \%$ & 28 \\
\hline$[32-40]$ & 36 & 116 & $18.0 \%$ & $58 \%$ & 36 \\
\hline$[40-48]$ & 29 & 145 & $14.5 \%$ & $72.50 \%$ & 44 \\
\hline$[48-56]$ & 28 & 173 & $14.0 \%$ & $86.50 \%$ & 52 \\
\hline$[56-64]$ & 17 & 190 & $8.5 \%$ & $95 \%$ & 60 \\
\hline$[64-72]$ & 5 & 195 & $2.5 \%$ & $97.50 \%$ & 68 \\
\hline$[72-80]$ & 4 & 199 & $2.0 \%$ & $99.50 \%$ & 76 \\
\hline$[80-88]$ & 1 & 200 & $0.5 \%$ & $100 \%$ & 84 \\
\hline
\end{tabular}

En este caso se aplicó el descriptor L2 a nivel 1, debido a que sólo se presenta el gráfico circular de torta representando las frecuencias relativas porcentuales de las categorías indicadas por números de 1 al 9 . Hay errores a considerar en la variable estadística consumo de agua, como variable cuantitativa discreta y, sobre todo, en el cambio débil de representación tabular a gráfico, no observando los intervalos de consumo de agua en el gráfico. La construcción adecuada de gráficos estadísticos es muy importante para comunicar información y estos pueden ser más eficaces que las tablas de resúmenes de datos. En este caso, estamos en presencia de un problema de cambio adecuado de una representación a otra (transnumeración), lo que dificulta la lectura, interpretación e integración de los datos del consumo de agua en el gráfico circular. Se sugirió a los estudiantes llevar a cabo un histograma.

Por otro lado, el grupo E aplicó el descriptor L2 a nivel 3 el tema de calidad de servicio de compañías telefónicas, recogiendo y tabulando una encuesta aplicada a 200 estudiantes sobre la percepción del servicio, declarando tres variables estadísticas: compañía de telefonía móvil que utiliza (variable cualitativa de tipo nominal), nivel de satisfacción que se tiene con la compañía telefónica (escala de 1 al 7, variable cuantitativa discreta) y el motivo de elec- 
ción de la compañía telefónica (económica o nivel de servicio, variable cualitativa nominal).

Del gráfico podemos extraer que de las tres compañías presentadas en dos de ellas (Entel y Movistar) se observa una clara inclinación a elegir, la compañía, por su servicio antes que por su economía; mientras tanto la compañía Claro es escogida por su economía más que por su servicio, siendo esta última compañía la que menor variación presenta en su preferencia a diferencia de Entel que presenta una mayor variación entre servicio y economía, siendo el primero de mayor elección.

Los estudiantes obtuvieron varios gráficos, siendo uno, el de barras agrupadas, (Figura 5) en que se muestra la relación entre las variables categóricas compañía telefónica utilizada y el motivo de su elección.

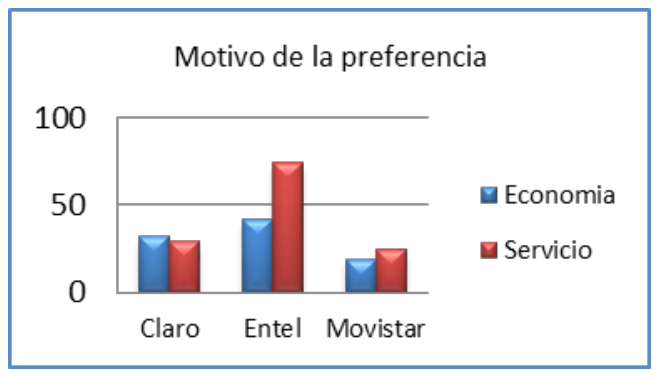

Figura 5. Satisfacción por las compañías telefónicas

\section{Reglas}

Los estudiantes utilizaron en su proyecto definiciones, propiedades y procedimientos, descritos en los siguientes descriptores:

D1. Las definiciones y propiedades son claras y correctas en este nivel educativo. Para analizar la información recogida de sus temas y aplicar el procedimiento de la estadística descriptiva, los estudiantes tuvieron que recurrir a las definiciones y propiedades de los conceptos de la unidad disponible en la plataforma virtual, tales como media, mediana, moda, varianza, desviación estándar, coeficientes de variación, de asimetría y curtosis. 
D2. Se presentan los procedimientos fundamentales de la estadística descriptiva. Los distintos grupos tuvieron la oportunidad de utilizar las definiciones y propiedades siguiendo el procedimiento de tabulación de datos, obtener representaciones gráficas, calcular medidas estadísticas y establecer relaciones entre variables.

D3. Se proponen situaciones que permitan generar definiciones, propiedades y procedimientos. Los estudiantes decidieron qué gráfico o medida de posición central es preferible para representar adecuadamente los datos, relacionaron las variables cuantitativas y generaron procedimientos según los objetivos planteados.

Tabla 7. Frecuencia (\%) del nivel de aplicación de los descriptores de reglas estadísticas

\begin{tabular}{c|c|c|c}
\hline & D1 & D2 & D3 \\
\hline Nivel 0 & $1(0,8 \%)$ & $0(0 \%)$ & $24(19,2 \%)$ \\
\hline Nivel 1 & $63(50,4 \%)$ & $51(40,8 \%)$ & $40(32 \%)$ \\
\hline Nivel 2 & $53(42,4 \%)$ & $66(52,8 \%)$ & $53(42,4 \%)$ \\
\hline Nivel 3 & $8(6,4 \%)$ & $8(6,4 \%)$ & $8(6,4 \%)$ \\
\hline
\end{tabular}

En la Tabla 7 se aprecia que 50,4\% de los estudiantes calcularon las medidas de posición (media, mediana, moda) y de dispersión (varianza, desviación estándar y coeficiente de variación). 42,4\% usan las medidas estadísticas y las interpretan según el contexto (D1 a nivel 2) y 6,4\% calculan e interpretan los coeficientes de asimetría y curtosis (D1 a nivel 3).

Respecto del procedimiento estadístico, descriptor 2, se observa que 40,8\% de los estudiantes realizaron correctamente la tabulación de información según la variabilidad de los datos. Hubo estudiantes que utilizaron la tabla de doble entrada de forma errónea, como muestra el siguiente ejemplo donde el grupo F resume las variables número del grupo familiar $X$ y gasto mensual en alimentos $Y$, por medio de una tabla bidimensional con muchos ceros en las celdas, lo que no es aconsejable para resumir y analizar la información.

De esta tabla se puede concluir fácilmente que de las 226 familias encuestadas, sólo 41 de ellas poseen 4 integrantes en su núcleo familiar y gastan entre $\$ 114.000$ y $\$ 200.000$ mensuales en alimentos. La covarianza es 102,55 con dependencia directa entre las variables. 
Tabla 8. Tabulación de datos

\begin{tabular}{|c|c|c|c|c|c|c|c|c|r|r|}
\cline { 2 - 13 } \multicolumn{1}{|c|}{} & $28-114$ & $114-200$ & $200-286$ & $286-372$ & $372-458$ & $458-544$ & $544-630$ & $630-716$ & $716-802$ & $\mathrm{ni}$ \\
\hline 1 & 5 & 0 & 0 & 0 & 0 & 0 & 0 & 0 & 0 & 5 \\
\hline 2 & 15 & 3 & 0 & 0 & 0 & 0 & 0 & 0 & 0 & 18 \\
\hline 3 & 15 & 22 & 2 & 1 & 1 & 0 & 0 & 0 & 0 & 41 \\
\hline 4 & 8 & 41 & 24 & 2 & 1 & 0 & 0 & 0 & 1 & 77 \\
\hline 5 & 7 & 16 & 35 & 5 & 0 & 2 & 0 & 0 & 0 & 65 \\
\hline 6 & 0 & 3 & 4 & 5 & 0 & 0 & 1 & 0 & 0 & 13 \\
\hline 7 & 0 & 0 & 3 & 0 & 1 & 1 & 0 & 0 & 0 & 5 \\
\hline 8 & 0 & 0 & 1 & 0 & 0 & 0 & 0 & 0 & 0 & 1 \\
\hline 9 & 0 & 0 & 0 & 0 & 0 & 0 & 0 & 1 & 0 & 1 \\
\hline n.j & 50 & 85 & 69 & 13 & 3 & 3 & 1 & 1 & 1 & 226 \\
\hline
\end{tabular}

De los participantes 52,8\% elaboraron gráficos adecuados para resumir sus datos. Muy pocos alcanzaron el descriptor de nivel 3, buscando relaciones entre las variables de estudio, dificultad también encontrada por Inzunza (2016). Un ejemplo es el grupo G, donde calcularon e interpretaron el coeficiente de correlación de las variables consumo de energía y gasto total en 220 familias, obteniendo una relación lineal fuerte entre ambas variables.

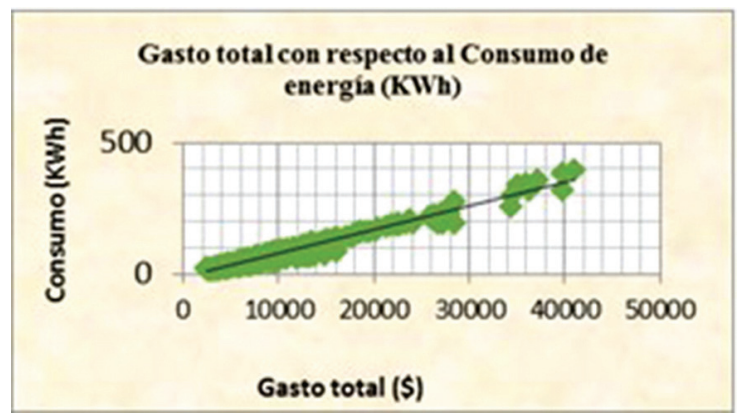

Figura 6. Relación entre el gasto y consumo de energía por familia 
También, hubo errores en la predicción del modelo de regresión lineal simple al confundir el error típico con el error aleatorio del modelo de regresión, como se muestra a continuación en las variables $Y$ consumo de luz y $X$ número de integrantes de la familia.

De la planilla Excel se obtuvo: B1=38.01220156, B0 $=140.1625359$ y error típico $=$ 26.80845918

Así podemos definir la ecuación de regresión lineal Y=140,16 +38,01 X+26,81

Por último, se presenta un ejemplo del descriptor D3 a nivel 2, el grupo H utilizó el siguiente gráfico que les permitió comparar las variables.

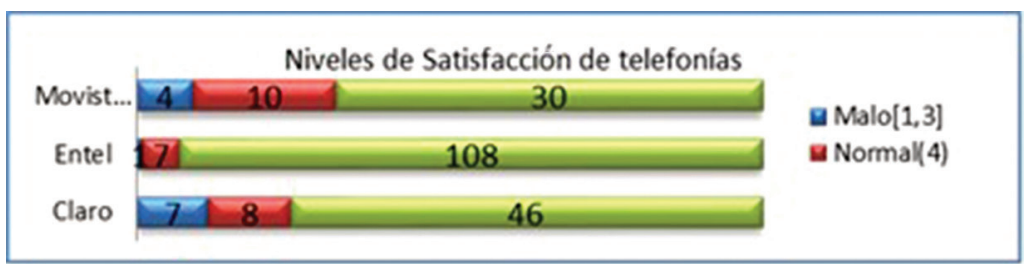

Figura 7. Satisfacción por las compañías telefónicas

\section{Argumentos}

Los estudiantes en la sección de conclusiones mencionaron los resultados más importantes obtenidos con base en el análisis estadístico de los datos. El diálogo producido en las sesiones de aula y tutoría fuera de clase contribuyó a la argumentación de los estudiantes en el procedimiento estadístico. En esta componente se desarrollaron dos descriptores:

A1. Las explicaciones, comprobaciones y demostraciones son adecuadas al nivel educativo.

A2. Se promueven situaciones donde el estudiante tenga que argumentar.

En las conclusiones de los proyectos los estudiantes presentaron una síntesis de comprensión e interpretación de la información estadística, mediante el uso 
correcto del conocimiento estadístico en dos contextos: la utilización de la metodología de estadística descriptiva y el uso de recursos informáticos.

Tabla 9. Frecuencia (\%) del nivel de aplicación de los descriptores, argumento estadístico

\begin{tabular}{c|c|c}
\hline & A1 & A2 \\
\hline Nivel 0 & $2(1,6 \%)$ & $2(1,6 \%)$ \\
\hline Nivel 1 & $57(45,6 \%)$ & $58(46,4 \%)$ \\
\hline Nivel 2 & $55(44 \%)$ & $59(47,2 \%)$ \\
\hline Nivel 3 & $11(5,6 \%)$ & $6(4,8 \%)$ \\
\hline
\end{tabular}

En la Tabla 9 se muestran los resultados de estos descriptores observando que la mitad de los estudiantes alcanzaron los niveles 2 y 3 , por tanto no fue fácil explicitar las argumentaciones en los procedimientos estadísticos. En el nivel terciario, escasamente, los estudiantes tienen la oportunidad de desarrollar el razonamiento estadístico, por lo general se promueve las demostraciones formales de los conceptos y propiedades.

A continuación, se presentan dos ejemplos donde se aplican los descriptores de nivel 2 y 3 . El grupo I aplica el descriptor D1 a nivel 2 a la situación de transporte urbano sin especificar la justificación de la afirmación.

Estudio del flujo promedio de transporte en un peaje de Concepción. Se analizaron dos variables independientes, autos de bajo tonelaje y alto tonelaje, a través de medidas de tendencia central y de dispersión, comprobado que la mayor frecuencia por el control de peaje corresponde a los autos de menor tonelaje.

Otro caso, fue el estudio de suelos que el grupo J aplica el descriptor D2 a nivel 3 , haciendo referencia a la necesidad de argumentar por medio de la comparación de las diferencias en las variables analizadas.

Los suelos de Santa Bárbara, localidad que no fue afectada por la ceniza volcánica, también contienen metales pesados en el mismo orden de magnitud que los encontrados en los suelos de Chaitén. En el metal de arsénico se observan concentraciones menores. 


\section{Relaciones}

El trabajo de proyectos, orientado a las ciencias de la ingeniería, concede la conexión con los procedimientos de análisis de datos y usos de gráficos y resúmenes estadísticos en los problemas propuestos. Los estudiantes pusieron a prueba el siguiente descriptor:

R1. Los objetos matemáticos (problemas, definiciones, propiedades, procedimientos, proposiciones, argumentos) se relacionan y conectan entre sí.

Tabla 10. Frecuencia (\%) del nivel de aplicación del descriptor de las relaciones

\begin{tabular}{c|c}
\hline & R1 \\
\hline Nivel 0 & $6(4,8 \%)$ \\
\hline Nivel 1 & $52(41,6 \%)$ \\
\hline Nivel 2 & $59(47,2 \%)$ \\
\hline Nivel 3 & $8(6,4 \%)$ \\
\hline
\end{tabular}

Se observa en la Tabla 10 que 46,4\% de los estudiantes del grupo curso no alcanzó el nivel 2; de éstos 4,8\% no logró identificar posibles relaciones entre los conceptos estadísticos. Por otro lado, 47,2\% consigue relacionar los objetos matemáticos pero no realiza argumentaciones correctas y contextualizadas, que sólo fue alcanzado por 6,4\%.

A continuación, se ilustra una situación problema sobre predicciones sismológicas presentada por el grupo K. Desarrollan el descriptor R1 a nivel 2. Se observa la interrelación de los objetos matemáticos conectando la secuencia del procedimiento de la estadística descriptiva, pero se equivocan en la interpretación del coeficiente de correlación.

Problema: Chile se encuentra en contacto con las placas de Nazca y Sudamericana, y en los últimos años se han intensificado los eventos sísmicos, algunos considerados réplicas del terremoto del 27 febrero del 2010. Nos interesa analizar si existe relación entre las variables magnitud en grados Richter y profundidad en kilómetros de los movimientos sísmicos, ¿Cuál es la magnitud promedio de los sismos? ¿Qué 
distribución de frecuencias tiene asociada la profundidad de los sismos? ¿Cuál de las dos variables magnitud y profundidad presenta mayor variabilidad?

Lenguaje: Un modelo de regresión lineal simple para las variables magnitud y profundidad de los sismos es $y=0,0043 x+3,0088$

Propiedades: El coeficiente de variación de los datos de magnitud de los sismos fue de $20.3 \%$, lo que indica que tiene una variabilidad de datos homogéneos ya que el CV es $\leq 35 \%$

Procedimientos: 74.79 metros fue la profundidad promedio de cada sismo; de los 214 registros de sismos, 50\% presenta, a lo más, una profundidad de 63.9 metros; 104.00 metros, es la profundidad que más se repite para los sismos durante un día registrado en las regiones de Chile. Podemos decir que no hay una simetría y que es confirmado por el coeficiente de asimetría de 1.15 que indica una distribución positiva sesgada a la derecha.

Argumentos: Chile es un país sísmico, en un día particular la magnitud promedio fue de 3.35 grados Richter con una profundidad media de 78.79 metros; es por esto que Chile debería poseer un buen sistema de precaución en caso de cualquier terremoto o sismo de gran intensidad. El análisis indicó que existe una correlación de 0,36 entre las variables permitiendo obtener un modelo matemático lineal para llevar a cabo tendencia y estimación de los sismos. (En esta interpretación la correlación es débil y por tanto no es aconsejable la predicción mediante un modelo lineal).

Una vez entregados los informes escritos de los proyectos, los estudiantes realizaron una presentación oral, mediante una selección aleatoria por grupo. La evaluación del proyecto consideró las calificaciones del docente (heteroevaluación) y la de pares por estudiantes (coevaluación). Las argumentaciones de los estudiantes se complementaron y debatieron en las exposiciones del proyecto, por medio de preguntas y comentarios del profesor respecto de los aciertos y errores presentes en los análisis de información. De esta manera se generó la institucionalización de las soluciones en las diversas situaciones problemas sugeridas por los estudiantes y que, además, fueron detallados en la calificación de los informes escritos.

En general, los proyectos fueron bien presentados, destacando la apropiación e interés de temas propios de la ingeniería, como el análisis químico de suelos 
y los caudales de ríos. La Tabla 11 muestra las calificaciones de docentes y estudiantes en las exposiciones; hubo un buen nivel en el discurso, fluidez de los temas y uso del lenguaje en el análisis de datos, alcanzando $87 \%$ de logro en la aplicación de los elementos metodológicos (RA1). Se obtuvo 85\% de aprobación en el uso adecuado del lenguaje en los proyectos. Todos los grupos usaron la informática (RA2). Se obtuvo $71 \%$ en la precisión de los objetivos propuestos en los proyectos.

Tabla 11. Pauta de valoración de las presentaciones de los proyectos

\begin{tabular}{l|c|c|c}
\hline \multicolumn{1}{c|}{ Criterio: Escala de valoración } & Puntaje ideal & Puntaje promedio & $\%$ \\
\hline 5.1 Contexto del problema & 04 & 3.85 & 96 \\
\hline 5.2 Claridad de los objetivos & 01 & 0.70 & 71 \\
$\quad$ de las hipótesis & 01 & 0.88 & 88 \\
\hline $\begin{array}{l}\text { 5.3 Aplicación del procedimiento de la } \\
\text { estadística descriptiva }\end{array}$ & 03 & 2.61 & 87 \\
\hline 5.4 Uso de los recursos informáticos & 02 & 2.00 & 100 \\
\hline $\begin{array}{l}5.5 \text { Uso del lenguaje en los resultados de la } \\
\text { estadística descriptiva }\end{array}$ & 03 & 2.55 & 85 \\
\hline 5.6 Presentación personal & 02 & 2.00 & 100 \\
\hline 5.7 Motivación e interés en el tema & 03 & 2.88 & 96 \\
\hline
\end{tabular}

Finalmente, 114 estudiantes completaron un cuestionario de autovaloración sobre la percepción de logro alcanzado en las distintas fases o descriptores del proyecto (ver Tabla 12). Los resultados indican una autovaloración positiva en las fases del proyecto, alcanzando mayoritariamente los niveles 2 ó 3, destacando los descriptores perseverancia y voluntad de logro, identificación y formulación del problema, representación e interpretación gráfica, declaración de variables, pensamiento creativo. Y comunica las posibilidades: pensamiento crítico e integración del conocimiento, uso de recursos informáticos y comunicación escrita. En cambio, los estudiantes, se sienten más débiles en las habilidades blandas de administración del tiempo y de los recursos, formación, crecimiento, evolución y liderazgo de equipos, búsqueda de información y formulación de hipótesis. 
Tabla 12. Autovaloración de los estudiantes en el desarrollo del proyecto según niveles en los descriptores

\begin{tabular}{|c|c|c|c|c|}
\hline \multirow[b]{2}{*}{ DESCRIPTORES } & \multicolumn{4}{|c|}{ NIVELES } \\
\hline & $\begin{array}{c}\text { Nivel } 0 \\
\text { Insuficiente }\end{array}$ & $\begin{array}{l}\text { Nivel } 1 \\
\text { Aceptable }\end{array}$ & $\begin{array}{l}\text { Nivel } 2 \\
\text { Bien }\end{array}$ & $\begin{array}{l}\text { Nivel } 3 \\
\text { Excelente }\end{array}$ \\
\hline Identificación y formulación del problema & 1 & 9 & 66 & 38 \\
\hline Búsqueda en la literatura impresa y electrónica & 9 & 29 & 42 & 34 \\
\hline Define los objetivos general y específicos & 1 & 20 & 38 & 55 \\
\hline Formulación de hipótesis & 5 & 19 & 48 & 42 \\
\hline Explicita las variables estadísticas & 2 & 12 & 39 & 61 \\
\hline Análisis cuantitativo & 2 & 19 & 62 & 31 \\
\hline $\begin{array}{l}\text { Representa e interpreta la información por } \\
\text { gráficos }\end{array}$ & 2 & 11 & 47 & 54 \\
\hline Solución y recomendaciones al problema & 2 & 20 & 59 & 33 \\
\hline $\begin{array}{l}\text { Coherencia entre objetivos, metodología y } \\
\text { resultados }\end{array}$ & 1 & 17 & 49 & 47 \\
\hline Uso de recursos informáticos & 1 & 16 & 44 & 53 \\
\hline Perseverancia, y voluntad de logro & 1 & 4 & 42 & 67 \\
\hline $\begin{array}{l}\text { Pensamiento creativo y comunica las } \\
\text { posibilidades }\end{array}$ & 1 & 13 & 58 & 42 \\
\hline $\begin{array}{l}\text { Pensamiento crítico e integración del } \\
\text { conocimiento }\end{array}$ & 2 & 14 & 61 & 37 \\
\hline Aprendizaje y educación durante el proyecto & 2 & 14 & 58 & 40 \\
\hline Administración del tiempo y de los recursos & 8 & 45 & 48 & 13 \\
\hline Formación de equipos efectivos & 17 & 20 & 40 & 37 \\
\hline Crecimiento y evolución de equipos & 12 & 21 & 42 & 39 \\
\hline Liderazgo de equipos & 8 & 18 & 45 & 43 \\
\hline Comunicación escrita & 3 & 14 & 52 & 45 \\
\hline Comunicación gráfica & 2 & 22 & 50 & 40 \\
\hline Presentaciones orales & 2 & 24 & 54 & 34 \\
\hline
\end{tabular}




\section{CONCLUSIONES}

Una síntesis de resultados de los descriptores analizados en las componentes de idoneidad epistémica es presentada (Figura 8) mediante valores medios obtenidos por los estudiantes. En términos de Godino (2009) es una valoración del conocimiento del contenido estadístico en los proyectos desarrollados. La gráfica muestra un conocimiento de estadística descriptiva no esperado para este nivel educativo, los estudiantes en promedio hicieron referencia a los descriptores pero no se centraron en el contenido estadístico (nivel 2).

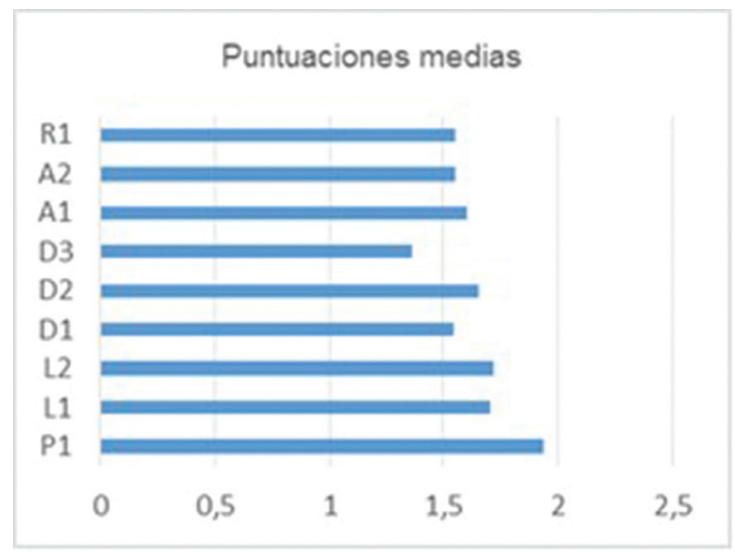

Figura 8. Puntuación media en los descriptores de idoneidad epistémica

En la asignatura de probabilidad y estadística se presentaron 45 proyectos de los cuales 93\% fueron aplicados al área de la ingeniería. Las dificultades encontradas en los estudiantes correspondieron a: la declaración de los objetivos, tabulación de datos en una y dos variables, interpretación de la información, uso adecuado de gráficos y medidas estadísticas, aplicación de propiedades y determinar relaciones entre variables. Coincidimos con Juárez e Inzunsa (2014) que la comprensión de conceptos estadísticos y su razonamiento no son procesos triviales y que para su desarrollo se requiere poner en juego competencias de mayor nivel cognitivo. Sin embargo, los proyectos aplicados a problemas reales han permitido a los estudiantes conjugar la apropiación del razonamiento estadístico, hacer una 
valoración crítica por pares en la comunicación de los proyectos, y aumentar la motivación y responsabilidad por su propio aprendizaje.

Consideramos que uno de los factores que incidieron en nuestros resultados es la consecuencia de una formación previa dogmática y descontextualizada, fomentada por el uso y memorización de fórmulas y procedimientos, que según Juárez e Inzunsa (2014), ayudan poco al desarrollo del razonamiento y del pensamiento estadístico de los estudiantes. En este sentido, Ben-Zvi y Garfield (2004) señalan que los estudiantes perciben la estadística como la matemática, tendiendo al cálculo, fórmulas y el procedimiento; en vez de desarrollar el razonamiento estadístico hacia la comprensión y capacidad de explicar e interpretar resultados de procesos estadísticos (Garfield, 2002).

En relación a los resultados de aprendizaje, podemos destacar la utilización de los elementos metodológicos de la estadística descriptiva (RA1). Gran parte de los estudiantes alcanzaron la formulación de un problema aplicado a la ingeniería, la recolección y análisis de datos reales y cumplieron con el formato establecido del proyecto de iniciación científica. La aplicación de la plataforma virtual, planillas de Excel y Power Point (RA2) aumentaron las posibilidades de trabajo y argumentaciones en el análisis de los problemas de ingeniería.

Finalmente, los estudiantes ampliaron el lenguaje y la argumentación, y se estableció la institucionalización de las soluciones en las múltiples situaciones de la ingeniería. Además, trabajaron colaborativamente con apoyo del docente, eligieron un tema a investigar, plantearon una problemática situada, recolectaron información y resumieron y analizaron los datos comunicando los resultados obtenidos. Hacemos notar que los profesores participantes han sido calificados en las categorías de muy bueno a bueno; en la encuesta de desempeño docente institucional.

\section{IMPLICACIONES PARA LA ENSEÑANZA}

El aprendizaje es un complejo proceso mediante el cual se asegura, en los estudiantes, el logro de habilidades y actitudes para su desarrollo integral. Consideramos que la metodología de proyectos favorece el aprendizaje de la estadística descriptiva a través de la planificación y realización de investigaciones, involucrando activamente a los estudiantes de ingeniería, cuando identifican un problema en contexto, plantean objetivos y variables estadísticas, obtienen representaciones gráficas con apoyo de tecnologías, analizan los datos y comunican una solución. 
La propuesta metodológica implementada ha permitido por un lado poner en acción y conjugar la triada conocimiento estadístico-contexto-análisis de datos. También, desarrollar en los estudiantes de ingeniería la habilidad de interpretar críticamente información estadística apoyada con recursos informáticos y comunicar adecuadamente de forma escrita, gráfica y verbal los resultados provenientes de sus análisis. Destacamos la motivación y autonomía de los estudiantes durante la ejecución del proyecto manifestada en la alta asistencia en el programa de actividad curricular tanto en el aula como el horario de consultas en oficinas de los docentes, además del cumplimiento en la entrega y comunicación del proyecto.

Uno de los objetivos de la enseñanza es promover que los estudiantes aprendan de manera autónoma y sean capaces de trabajar en equipo. Por medio de la estrategia activa efectuada permitió a los docentes la retroalimentación oportuna durante el proceso de la evaluación de los resultados de aprendizaje, es decir, dar importancia al retorno de la información o feedback después de haber realizado la evaluación (Morales, 2012).

En síntesis, plantemos que el aprendizaje de la estadística por proyectos es un medio para promover (1) la evaluación del conocimiento estadístico, donde los estudiantes son sujetos activos de aprendizaje en el contexto de ciencia y sociedad; (2) la utilidad del computador para ofrecer representaciones diferencias hacia una comprensión global del significado de conceptos y propiedades; (3) ahondar en las ideas estadísticas fundamentales; (4) la necesidad de un cambio importante en la concepción de la enseñanza de la matemática y la ciencia en el nivel terciario; (5) la reflexión sobre la formación del docente en educación en ingeniería, en términos de Porlán et al. (2010) conveniencia de que los docentes construyan un conocimiento práctico profesional, (6) una actitud positiva hacia la enseñanza y aprendizaje de la estadística.

Este trabajo de diseño e implementación sobre estadística descriptiva y su aprendizaje por proyectos puede contribuir para estudios más amplios, según nivel educativo, sobre proyectos de tipo experimentales, con mayor duración de tiempo y considerando temas de inferencia estadística sobre intervalos de confianza y pruebas de hipótesis.

Finalmente, debemos reforzar las competencias estadísticas y resultados de aprendizaje para la formación óptima e integral del profesional ingeniero que se espera egrese hoy de las Escuelas de Ingeniería. Para ello, podríamos avanzar en estudios sobre dinámicas de cambios de los docentes en los procesos de enseñanza de las ciencias básicas y ciencias de la ingeniería. 


\section{REFERENCIAS}

Alvarado, H., y Batanero, C. (2007). Dificultades de comprensión de la aproximación normal a la distribución binomial. Números, 67.

Alvarado, H., y Segura, N. (2012). Significado de las distribuciones muestrales en textos universitarios de Estadística. Revista electrónica de investigación en educación en ciencias, 7(2), 54-71.

Alvarado, H., Galindo, M., y Retamal, L. (2013). Comprensión de la distribución muestral mediante configuraciones didácticas y su implicación en la inferencia estadística. Enseñanza de las Ciencias, 31(2), 1-17.

Arteaga, P., Batanero, C., Cañadas, G., y Gea, M. M. (2012). Evaluación del conocimiento especializado de la estadística en futuros profesores mediante el análisis de un proyecto estadístico. Educ. Matem. Pesq., 14(2), pp. 279-297.

Batanero, C., Burrill, G., y Reading, C. (2011) (Eds). Teaching statistics in school mathematics. Challenges for teaching and teacher education: A Joint ICMI/IASE Study. Berlin: Springer.

Batanero, C., y Díaz, C. (2011). Estadística con proyectos. Granada, Universidad de Granada.

Batanero, C., Díaz, C., Contreras, J. M., y Roa, R. (2013). El sentido estadístico y su desarrollo. Números, 83, 7-18.

Ben-Zvi, D., y Garfield, J. (2004). Statistical Literacy, Reasoning, and Thinking: Goals, definitions, and challenges. En D. Ben-Zvi y J. Garfield (eds.), The Challenge od Developing Statistical Literacy, Reasoning, and Thinking (pp. 3-15), Netherlands, Kluwer Academic Publishers.

Burrill, G., y Biehler, R. (2011). Fundamental statistical ideas in the school curriculum and in trainingteachers. En C. Batanero, G. Burrill y C. Reading (Eds.), Teaching statistics in school mathematics. Challenges for teaching and teacher education - A joint ICMI/ IASE study (pp. 57-69). Dordrecht: Springer.

Carvalho, C. (2001). Interaçao entre pares. Contributos para a promoçao do desenvolvimiento lógico e do desempenho estatístico no 7o ano de escolaridade. PhD. University of Lisbon.

Chance, B., delMas, R., y Garfield, J. (2004). Reasoning about Sampling Distributions. En Dani Ben-Zvi y Johan Garfield (eds.), The Challenge od Developing Statistical Literacy, Reasoning, and Thinking (pp. 295-323), Netherlands, Kluwer Academic Publishers,

Cobo, B., y Batanero, C. (2004). Significado de la media en los libros de texto de secundaria. Enseñanza de las ciencias 22(1), 5-18. 
Estrella, S., y Vidal-Szabó, P. (2017). Alfabetización estadística a través del Estudio de Clase: representaciones de datos. UNO, Revista de Didáctica de las Matemáticas, 78, 12-17.

Flores, A., y Pinto, J. (2017). Características de la enseñanza de la estadística por proyectos. Acta Latinoamericana de Matemática Educativa, vol. 30, 263-271.

Gal, I. (2002). Adult's statistical literacy. Meaning, components, responsabilities. International Statistical Review, 70(1), pp. 1-25.

Garfield, J. (2002). The challenge of developing Statistical Reasoning. Journal of Statistics Education, 10 (3). Consultado el 02 de marzo de 2017 en:

http://www..amstat.org/publications/jse/v10n3/garfield.html

Gil, A. (2010). Proyectos de Estadística en Primaria. Material editado por el Instituto Canario de Estadística (ISTAC). Números, 75, 21-129.

Godino, J. D. (2009). Categorías de análisis de conocimientos del profesor de matemáticas. Unión, 20, 13-31.

Godino, J. D., Batanero, C. (1997). Clarifying the meaning of mathematical objects as a priority area of research in mathematics education. En J. Kilpatrick \& A. Sierpinska (Eds.), Mathematics education as a research domain: A search for identity, (pp. 177196). Dordrecht Kluwer.

Godino, J. D., Giacomone, B., Batanero, C., y Font, V. (2017). Enfoque ontosemiótico de los conocimientos y competencias del profesor de matemáticas. Bolema, 31(57), 90-113.

Groth, R. E., y Bergner, J. A. (2006) Preservice elementary teachers' conceptual and procedural knowledge of mean, median, and mode. Mathematical Thinking and Learning, 8, 37-63.

Inzunza, S. (2016) Análisis de datos bivariados en un ambiente basado en applets y software dinámico. Educación Matemática, 28(3), 61-89.

Juárez, J., e Inzunsa, S. (2014). Comprensión y razonamiento de profesores de matemáticas de Bachillerato sobre conceptos estadísticos básicos. Perfiles Educativos, XXXVI (146), 14-29.

Letelier, M., López, L., Carrasco, R., y Pérez, P. (2005). Sistema de competencias sustentables para el desempeño profesional en ingeniería. Rev. Fac. Ing., 13(2), 91-96.

MacGillivray, H., y Pereira-Mendoza, L. (2011). Teaching statistical thinking through investigative projects. En C. Batanero, G. Burrill, y C. Reading (Eds.), Teaching

statistics in school mathematics. Challenges for teaching and teacher education. A joint ICMI and IASE study (pp. 109-120). New York: Springer.

Martín, P. (2006). Achieving success in industrial training. En A. Rossman y B. Chance (Eds.), Proceedings of ICOTS-7. Salvador (Bahia): International Association for Statistical Education. CDROM. Consultado el 29 de abril de 2017 en: http://iase-web.org/ documents/papers/icots7/4A2 MART.pdf 
Mayén, S., Batanero, C., y Díaz, C. (2009). Conflictos semióticos de estudiantes mexicanos en un problema de comparación de datos ordinales. Revista Latinoamericana de Investigación en Matemática Educativa, 12(2), 151-178.

Mayén, S., Cobo, B., Batanero, C., y Balderas, P. (2007). Comprensión de las medidas de posición central en estudiantes mexicanos de bachillerato. Unión, Revista Iberoamericana de Educación Matemática, 9, 187-201.

Morales, P. (2012). La información de retorno en la evaluación (feedback), Torre Puente, Juan Carlos. Educación y nuevas sociedades, (191-220). Madrid: Universidad Pontificia Comillas.

Muñoz, M., Martínez, C., Cárdenas, C., y Cepeda, M. (2013). Active Learning in first-year Engineering courses at Universidad Católica de la Santísima Concepción, Chile. Australasian Journal of Engineering Education, 19(1), 27-38.

Porlán, R., Martín del Pozo, R., Ribero, A., Harres, J., Azcárate, P., y Pizzato, M. (2010). El cambio del profesorado de ciencias I: Marco teórico y formativo. Enseñanza de las Ciencias, 28(1), 31-46.

Retamal, L., Alvarado, H., y Rebolledo, R. (2007). Understanding of sample distributions for a course on statistics for engineers. Ingeniare, 15, 6-17.

Romeu, J. (2006). Teaching engineering statistics to practicing engineers. En A. Rossman y B. Chance (Eds.), Proceedings of ICOTS-7. Salvador (Bahia): International Association for Statistical Education, CDROM. Consultado el 29 de abril de 2017 en: http://iaseweb.org/documents/papers/icots7/4A1 ROME.pdf

Sánchez, E., y Hoyos, V. (2013). La estadística y la propuesta de un currículo por competencias. En: A. Salcedo (Ed.), Educación Estadística en América Latina: Tendencias y perspectivas (pp. 211-227), Universidad Central de Venezuela. ISBN: 978-980-00-2744-8.

Watson, J. (2006). Statistical literacy at school: growth and goals. Mahwah, NJ: Lawrence Erlbaum Associates.

Wild, C. J., y Pfannkuch, M. (1999). Statistical thinking in empirical enquiry. International Statistical Review, 67(3), 223-265.

\section{Hugo Alejandro Alvarado MartíneZ \\ Domicilio: Roberto Ovalle 50 casa 16 Penco, Chile \\ Código postal: 4150000}

Teléfono institucional: 56412345686 